\title{
Registros relativos à contracepção em prontuários de mulheres atendidas por abortamento no Hospital Universitário de Londrina - Paraná, de 2001 a 2005
}

\author{
Records related to contraception in hospital records of abortion \\ patients hospitalized in the University Hospital of Londrina - \\ Paraná, 2001 - 2005
}

\author{
Joice de Souza Jardim ${ }^{1}$; Marta Lúcia de Oliveira Carvalho
}

Resumo

\begin{abstract}
No Brasil, o abortamento é a terceira causa de mortalidade materna. A melhoria da qualidade da assistência às mulheres atendidas por abortamento nos hospitais inclui acolhimento e aconselhamento sobre métodos contraceptivos, objetivando diminuir o risco de novos abortamentos, e essas ações podem ser desenvolvidas por qualquer dos membros da equipe de saúde. Este estudo, transversal e descritivo, analisou os registros relativos à contracepção em prontuários de mulheres atendidas por abortamento em Hospital Universitário de Londrina-PR, de 2001 a 2005. Em 46,8\% dos casos, o abortamento ocorreu em mulheres na faixa etária de 20 a 29 anos e a ocupação mais referida nos prontuários foi "trabalho doméstico não remunerado" (15\%); 75,7\% descreviam a existência de apenas um abortamento; em $94,1 \%$ não há referência ao abortamento ter sido provocado; em $76,2 \%$ dos prontuários não há registro relativo à contracepção pós-abortamento, evidenciando a necessidade de capacitação da equipe tanto para a qualificação da assistência quanto para prevenção de novos abortamentos.
\end{abstract}

Palavras-chave: Abortamento. Contracepção. Saúde da Mulher. Saúde Reprodutiva.

\begin{abstract}
In Brazil, the abortion is the third reason of maternal mortality. The improviment of health care quality to women attended for abortion relief in hospitals includes shelter and advising about contraceptive methods, looking for the decrease of chances of new abortions, activities that may be developed by anyone of health's team member. This transversal and descriptive research, proposes to analyze the concerning observations of contraception in records about women attended for abortion in the University Hospital of Londrina - Paraná 2001 - 2005. In 46,8\% of the study cases, the abortion occurred in women beetween 20 and 29 years old; most of them referred "domestic work non-paid" occupation (15\%); $75,7 \%$ describes the existence of only one abortion; there isn't any reference about induced abortion in $94,1 \%$ of the cases; In $76,2 \%$ of the hospital's records there is no information related to contraception after abortion, making clearly theneed of capacitating actions from the team concerning about the qualification of assistance and prevention of new abortions.
\end{abstract}

Key words: Abortion. Contraception. Women's Health. Reproductive's Health.

\footnotetext{
1. Aluna do Curso de Graduação em Enfermagem da Universidade Estadual de Londrina. E-mail: joice_souzaj@yahoo.com.br

2 Professora Adjunta do Departamento de Enfermagem da Universidade Estadual de Londrina, Doutora em Saúde Pública. E-mail: marta-londrina@uol.com.br
} 


\section{Introdução}

O Ministério da Saúde conceitua "abortamento" como o "expulsão ou extração de um produto da concepção, sem sinais de vida, com menos de 500 gramas e/ou estatura menor ou igual a $25 \mathrm{~cm}$ ou menos de 22 semanas de gestação". Já "aborto" é conceituado como "o produto da concepção expulso no abortamento" (BRASIL, 2007, p.16).

O abortamento é considerado crime, segundo o Código Penal Brasileiro, salvo os casos em que a vida da gestante corre perigo ou a gravidez é decorrente de estupro. A penalidade para as mulheres que se submetem ao abortamento de forma ilegal varia de 1 a 10 anos de prisão, com a pena duplicada para aqueles que o praticam ou o auxiliam (Código Penal Art. 126 - 129. Decreto Lei $n^{0}$ 2848). Entretanto, nota-se que, apesar das restrições legais existentes no país, os casos de abortamentos induzidos levados a julgamentos são raros (OSIS et al., 1996) e as restrições legais têm relação direta com o aumento da incidência de abortamento inseguro (WORLD HEALTH ORGANIZATION, 2004).

É impossível quantificar com precisão os índices de abortamento entre as mulheres brasileiras, devido à falta de notificação de casos admitidos em hospitais públicos e privados, e isso dificulta o cálculo dos índices reais de mortalidade materna (LIMA, 2000; PARPINELLI et al., 2000; LANGER, 2002; WORLD HEALTH ORGANIZATION, 2004). Um estudo da subnotificação de dados acerca da mortalidade materna apontou que $71,5 \%$ destas subnotificações estão relacionadas ao abortamento (PARPINELLI et al., 2000). Segundo o Ministério da Saúde, a cultura, a religião e as leis reprimem aquelas mulheres que desejam declarar seus abortamentos, impedindo a avaliação real do problema (BRASIL, 2005).

A discriminação por razões culturais, religiosas e legais também têm dificultado a atenção às complicações causadas por abortamentos inseguros. No Brasil, essas complicações ocupam o terceiro lugar entre as causas de mortalidade materna (BRASIL, 2001), mas em outros países elas são responsáveis pela maioria das mortes em idade reprodutiva (SALTER et al., 1997). A alta letalidade dessas complicações são decorrentes da falta de segurança e de qualidade da assistência prestadas a essas mulheres (LANGER, 2002; BRASIL, 2005). Segundo The Alan Guttmacher Institute (2007), a cada ano, cerca de 5 milhões de mulheres que realizam abortamentos inseguros são internadas por complicações. Entre elas, as mais comuns são hemorragias, infecções, perfurações, febre e infertilidade (SALTER et al., 1997; MURTA et al., 2001; LANGER, 2002; BRASIL, 2005; GUTTMACHER INSTITUTE, 2007). No Brasil, são gastos em torno de 45 milhões de reais anualmente para o tratamento das complicações do abortamento (BRASIL, 2005).

Os gastos com a assistência às complicações do abortamento já foram maiores, provavelmente devido à mudança no método abortivo mais adotado (ARILHA; BARBOSA, 1993). Em países desenvolvidos, ou em casos de mulheres com maior poder aquisitivo, o abortamento, mesmo sendo realizado por médicos, através de curetagens, poderiam causar complicações (MARTINS et al., 1991; HENSHAW; SING; HAAS, 1999). Em países em desenvolvimento, ou em casos em que as mulheres têm baixo poder aquisitivo, muitas realizavam o abortamento sozinhas ou com ajuda de parteiras ou curiosas, geralmente em condições inseguras, usando métodos que traziam grande risco de complicações pós-abortamento, como a introdução de objetos ou de líquidos cáusticos na vagina, a ingestão de chás, drogas ou outras substâncias e a massagem abdominal vigorosa (MARTINS et al., 1991; FONSECA et al., 1996; OSIS et al., 1996; HENSHAW; SING; HAAS, 1999).

Atualmente, ao buscar um método não invasivo e com menos riscos à saúde, as mulheres têm utilizado o misoprostol (ARILHA; BARBOSA, 1993; FONSECA et al., 1996; FONSECA et al., 
1998). Esta substância é utilizada no tratamento de úlceras gástricas e duodenais e contra-indicada em casos de gravidez, já que induz o abortamento por produzir contrações uterinas (ARILHA; BARBOSA 1993; HARVEY; CHAMPE; MYCEK, 1998).

Embora a modificação dos métodos abortivos venha provocando menos danos à saúde das mulheres e, provavelmente, esteja influenciando a queda da mortalidade por abortamento, é importante que a prevenção do abortamento seja incrementada, por meio da informação e o acesso facilitado aos métodos contraceptivos. Osis et al. (1999) entrevistaram mulheres a respeito do conhecimento dos métodos contraceptivos, e observaram que muitas os conhecem apenas de "ouvir falar". Provavelmente, se novos estudos avaliassem a qualidade da informação das mulheres acerca da contracepção, poderiam ser detectados conceitos errados acerca dos métodos.

Segundo Langer (2002), a falta de informação é o maior empecilho ao uso adequado dos contraceptivos. No Brasil, mais de 3 milhões de mulheres em idade reprodutiva necessitam de informações sobre a contracepção adequada, tanto para limitar como para espaçar os nascimentos de filhos (TAVARES; LEITE; TELLES, 2007) e a maior escolaridade e melhor classificação socioeconômica associam-se a um maior escore de conhecimento sobre contracepção (ESPEJO et al., 2003).

Segundo estimativas da Organização Mundial da Saúde (WORLD HEALTH ORGANIZATION, 2004), de cada cinco gestações, duas resultam da não utilização de anticoncepcionais, da falha ou do uso inadequado dos mesmos. Três pesquisas realizadas no Brasil, estudando que porcentagem das mulheres que abortaram não usavam contracepção quando ficaram grávidas, apontam situação similar: Martins et al. (1991), em dados colhidos em favela do Rio de Janeiro entre 1984 e 1985, encontraram 62,3\%; Fonseca et al. (1996), em pacientes atendidas em Hospital em Fortaleza, encontraram 61,1\%; Fonseca et al. (1998), em pacientes atendidas em hospital de Florianópolis entre 1992 e 1993, encontraram 53,9\%. Um estudo das complicações no pós-abortamento aponta que mais de dois terços das mulheres na América Latina, Ásia e África, que receberam atendimento por complicações de abortamento em condições de risco, não tinham usado métodos anticoncepcionais modernos alguma vez na sua vida (SALTER et al., 1997).

Sedgh et al. (2007), em estudo sobre contracepção nos países em desenvolvimento, apontaram que as razões pelas quais as mulheres não usam métodos contraceptivos, em sua maioria são: a preocupação acerca de seus efeitos colaterais, as dúvidas sobre suas conseqüências para a saúde e a inconveniência dos métodos. Entre as mulheres casadas, os motivos mais referidos se relacionaram com a crença de que não ficarão grávidas porque estão amamentando ou porque não têm relações sexuais freqüentemente. Esta última foi a maior razão referida entre as mulheres que nunca se casaram, assim como a idéia de que não deveriam ou não necessitariam utilizar algum método até o casamento. Para Tavares, Leite e Telles (2007), muitas vezes a utilização inadequada dos métodos pode relacionar-se com a insatisfação das usuárias e com o acesso restrito aos mesmos.

A orientação da contracepção tem inserção importante na assistência pós-abortamento, incluindo a distribuição regular de métodos, incluindo a contracepção de emergência, em um contexto de bom nível de comunicação entre a equipe de saúde e a paciente. Também inclui necessariamente o encaminhamento a serviços da rede básica para a continuidade da assistência em planejamento familiar (BRASIL, 2001; CONSÓRCIO DE ATENÇÃO PÓS-ABORTO, 2002; ORGANIZACIÓN MUNDIAL DE LA SALUD, 2003; BRASIL, 2005). 
É importante salientar que a mulher que aborta poderá engravidar em torno de 15 dias após o abortamento (BRASIL, 2001; BRASIL, 2005). Em pesquisas acerca das complicações de abortamento, foi observada uma grande incidência de abortamentos anteriores, como 45,9\% (SCHOR, 1990), 34\% (FONSECA et al., 1996), 48,3\% (FONSECA et al., 1998), 24,1\% (MURTA et al., 2001). Esses dados reforçam a orientação contraceptiva como parte indispensável da assistência ao pós-abortamento (SALTER et al., 1997; BRASIL, 2001).

A equipe de saúde deve exercer uma assistência mais qualificada a estas mulheres, a qual depende, fundamentalmente, da comunicação, e inclui o suporte emocional, o esclarecimento das possíveis dúvidas relacionadas ao abortamento, ciclo reprodutivo, DST's, violência sexual e a discussão do planejamento familiar (BRASIL, 2001; CONSÓRCIO DE ATENÇÃO PÓS-ABORTO, 2002; BILLINGS; VERNON, 2007).

O enfermeiro deve ser capaz de estabelecer um bom nível de comunicação, pois esta é uma ação terapêutica na recuperação da saúde (WEHBE; GALVÃO, 2001; OLIVEIRA et al., 2005), e também lhe cabe promover o relacionamento interpessoal, o suporte à tomada de decisão e a própria competência clínica. Porém, por ser sujeito de várias atribuições, em muitos momentos as atividades administrativas do enfermeiro acabam por sobressair às demais, afastando o profissional da assistência direta aos pacientes (WEHBE; GALVÃO, 2001). Estudos referentes às práticas de enfermagem nos diversos setores do hospital evidenciam a necessidade da reflexão e valorização da prática assistencial prestada pelos grupos de enfermeiros nos diferentes serviços (WEHBE; GALVÃO, 2001; PÁDUA, 2002; HADDAD, 2004; OLIVEIRA et al., 2005; DRAGANOV; REICHERT, 2007).

A assistência prestada ao paciente pode ser avaliada através dos registros em prontuários, pois o registro da assistência prestada tem uma finalidade legal, ética e administrativa para avaliar a qualidade e a continuidade da assistência global (PÁDUA, 2002; HADDAD, 2004; DRAGANOV; REICHERT, 2007).

Embora de grande importância para a assistência, comunicação e pesquisa, o prontuário do paciente muitas vezes tem sido preenchido de maneira inadequada, às pressas, tendo, como principais características, a ilegibilidade e a falta de dados objetivos e completos (CONSELHO REGIONAL DE MEDICINA DO DISTRITO FEDERAL, 2006).

Especificamente nos casos de abortamento, a imprecisão dos dados é um problema a ser considerado. Loureiro e Vieira (2004), ao entrevistar os médicos dos serviços de emergência de Ribeirão Preto (SP) acerca das anotações da suspeita de abortamento induzido no prontuário, observaram que a maioria deles $(63 \%)$ acredita que esta não deva ser anotada para não comprometer legalmente a paciente. Os autores afirmam que isso ocorre pelo desconhecimento acerca da lei do segredo profissional, a qual estabelece que é vedado ao médico "revelar fato de que tenha conhecimento em virtude do exercício de sua profissão, salvo por justa causa, dever legal ou autorização expressa do paciente" (Código de Ética Médica - Cap. IX, artigo 102) (LOUREIRO; VIEIRA, 2004).

Apesar de não terem sido localizados estudos específicos sobre a assistência de enfermagem no pós-abortamento, alguns trabalhos discutem a falta de qualidade dos registros de enfermagem em prontuário, os quais apontam para registros voltados apenas para as funções biológicas (PÁDUA, 2002; HADDAD, 2004; LABBADIA; ADAMI, 2004; DRAGANOV; REICHERT, 2007).

Para que haja a validação da assistência, é fundamental que toda a prática seja representada através de relatórios e registros que devem estar pautados em vocabulário científico, precisão e exatidão, para que se possa manter uma 
comunicação entre os membros da equipe de saúde (OLIVEIRA et al., 2005). Assim, para uma avaliação da assistência prestada no pósabortamento, seria necessário o estudo dos registros através de estudo dos prontuários das mulheres atendidas.

Desta forma, este trabalho tem o objetivo de analisar os registros de atendimentos ou orientações relativos à contracepção em prontuários de mulheres atendidas por abortamento no Hospital Universitário Regional do Norte do Paraná, de 2001 a 2005.

\section{Material e métodos}

Estudo descritivo, transversal, realizado em um Hospital Universitário localizado em LondrinaPR, principal referência municipal para os casos de abortamento e campo de formação de recursos humanos em saúde. O hospital constitui um campo de prática de cursos de graduação em Medicina, Enfermagem e outros, em que se poderia esperar a ocorrência de registros precisos e completos acerca da assistência prestada.

Foram objeto de estudo as anotações em prontuários de mulheres atendidas nos anos de 2001 a 2005, com diagnósticos referentes aos diversos códigos CID-10, relacionados aos diferentes tipos de abortamento. Foram excluídos os casos classificados como mola hidatiforme, gravidez ectópica, placenta prévia. Não foram excluídos os casos classificados como abortamento espontâneo, considerando a dificuldade de diferenciação entre estes casos e os de abortamento induzido pelo Misoprostol. Foram confrontadas as listagens de "curetagens pós-abortamento" com a de "ameaça de abortamento" para localizar eventuais registros incompletos ou incorretos. Com mulheres que, no período considerado para este estudo, tiveram registro em seu prontuário de mais de um episódio de abortamento, cada episódio recebeu uma entrada de dados específica.
A partir dos dados sobre o total de atendimentos por abortamento realizados no Hospital Universitário de 2001 a 2005, fornecidos pelo SAME, foi calculado o tamanho da amostra em 408 prontuários, considerando o erro amostral 0,05 e o intervalo de confiança $95 \%$.

Foi utilizado um formulário com questões fechadas, considerando variáveis relativas à caracterização sócio-demográfica e de vida reprodutiva da paciente atendida: Idade; Escolaridade; Estado marital; Cor; Profissão; Número de gestações; Número de filhos vivos; Número de abortamentos (incluindo o atual); Referência a ter sido provocado; Registro de algum tipo de orientação acerca de métodos anticoncepcionais.

Foi feito o estudo descritivo de todas as variáveis por meio de distribuição de freqüência absoluta e relativa. Os valores foram aproximados para 1 casa decimal.

Pesquisas sobre o abortamento necessitam excepcional atenção para a necessidade de confidencialidade dos dados e anonimato das pacientes cujos prontuários serão objeto de estudo. Nomes ou outras formas de identificação das mulheres cujos prontuários foram analisados não constaram de relatórios parciais ou finais da pesquisa, assim como dos profissionais de saúde cujas anotações e registros foram pesquisados.

O projeto foi analisado e aprovado pelo Comitê de Ética em Pesquisa envolvendo seres humanos da Universidade Estadual de Londrina, tendo recebido autorização do Diretor-Superintendente para sua realização no Hospital Universitário Regional do Norte do Paraná.

\section{Resultados e Discussão}

Os dados sócio-demográficos registrados nos 408 prontuários analisados de mulheres atendidas por abortamento estão apresentados na Tabela 1 . 
Tabela 1. Dados sócio-demográficos registrados em prontuários de mulheres atendidas por abortamento no Hospital Universitário de Londrina - Paraná, de 2001 a 2005.

\begin{tabular}{|c|c|c|}
\hline Idade & Freqüência & Porcentagem \\
\hline até 15 anos & 11 & $2,7 \%$ \\
\hline 15 a 19 & 69 & $16,9 \%$ \\
\hline 20 a 24 & 97 & $23,8 \%$ \\
\hline 25 a 29 & 94 & $23,0 \%$ \\
\hline 30 a 34 & 64 & $15,7 \%$ \\
\hline 35 a 39 & 45 & $11,0 \%$ \\
\hline 40 e mais & 25 & $6,1 \%$ \\
\hline não há registro & 3 & $0,7 \%$ \\
\hline \multicolumn{3}{|l|}{ Estado Marital } \\
\hline unida (casada, amasiada) & 182 & $44,6 \%$ \\
\hline não unida (solteira, separada, viúva) & 166 & $40,7 \%$ \\
\hline não especificado & 60 & $14,7 \%$ \\
\hline \multicolumn{3}{|l|}{ Raça } \\
\hline branca & 343 & $84,1 \%$ \\
\hline negra & 25 & $6,1 \%$ \\
\hline parda & 3 & $0,7 \%$ \\
\hline asiática & 1 & $0,2 \%$ \\
\hline não especificado & 36 & $8,8 \%$ \\
\hline \multicolumn{3}{|l|}{ Profissão ocupação } \\
\hline "do lar" & 61 & $15,0 \%$ \\
\hline empregada serv/industr/comerc & 31 & $7,6 \%$ \\
\hline estudante & 13 & $3,2 \%$ \\
\hline doméstica assalariada & 12 & $2,9 \%$ \\
\hline autônoma & 6 & $1,5 \%$ \\
\hline não especificado & 285 & $69,9 \%$ \\
\hline \multicolumn{3}{|l|}{ Procedência } \\
\hline Londrina & 385 & $94,4 \%$ \\
\hline outra cidade & 21 & $5,1 \%$ \\
\hline não há registro & 2 & $0,5 \%$ \\
\hline Total & 408 & $100,0 \%$ \\
\hline
\end{tabular}


Houve predominância da faixa etária de 20 a 29 anos, como mostra a Tabela 1. Pode-se inferir que, como a idade reprodutiva segue este padrão, há maior possibilidade da ocorrência de abortamentos neste período da vida. Resultados equivalentes podem ser encontrados em estudos como os de Schor (1990), Fonseca et al. (1996; 1998), Sorrentino e Lebrão (1998), Murta et al. (2001) e Cohen (2007). É importante ressaltar a considerável ocorrência de adolescentes (abaixo de 20 anos) nesta amostra, sendo elas responsáveis por $19,6 \%$ do total. O Ministério da Saúde também destacou o grande percentual de adolescentes internadas por abortamento em 1998, segundo dados do SIH/SUS, as quais representavam $22,5 \%$ da população analisada. Peres e Heilborn (2006), em estudo multicêntrico sobre a cogitação e a prática do abortamento na adolescência, discutiu as barreiras existentes no país para controle da reprodução, e reforçou que os adolescentes brasileiros passam por dificuldades no acesso aos contraceptivos e aos serviços de saúde, além da falta de oportunidades sociais, educacionais e profissionais que nem sempre aparecem e contribuem para a ocorrência de gestações indesejadas e, conseqüentemente, de abortamentos.

Em relação ao estado marital, não houve predominância entre as categorias unida e não unida, resultado semelhante ao encontrado por Schor (1990). Houve variação do item "estado marital" em diversos estudos: alguns apontam predominância de mulheres não unidas (HARDY et al., 1994; COSTA et al., 1995; FONSECA et al., 1996; MOTTA, 2005; COHEN, 2007) e outros, de mulheres unidas (MARTINS, 1991; FONSECA et al., 1998). Segundo o Guttmacher Institute (2007), é maior o número de mulheres unidas que não utilizam os métodos anticoncepcionais comparadas às não unidas e, conseqüentemente, uma maior chance da ocorrência de uma gestação indesejada, que pode levar ao abortamento.

Neste estudo, verificou-se a predominância de mulheres da raça branca. Porém, Cohen (2008), em um estudo da relação entre abortamento e raça, afirma que é maior a proporção de abortamentos entre mulheres negras. Nos EUA, ela pode chegar até a ser 5 vezes maior comparado aos abortamentos entre mulheres brancas. Este dado está relacionado às dificuldades das mulheres de comunidades menos favorecidas em receber uma assistência de qualidade, que ofereça métodos contraceptivos com orientações precisas.

Há que se ressaltar que, no serviço em que a pesquisa foi realizada, a classificação de raça a que pertence a paciente é feita pelo próprio profissional que a admite, podendo haver variação entre critérios. Além disso, a definição de raça no Brasil é uma questão complexa e delicada, tendo em vista a miscigenação da população (HOFFMAN; CENTENO, 2006).

Em muitas situações, a profissão/ocupação pode ser a causa do abortamento, considerandose os riscos ou esforços aos quais as mulheres podem se submeter para executar as tarefas inerentes. A natureza da relação de trabalho, com ou sem vínculo de contrato e, por conseqüência, com ou sem amparo de previdência social, pode ser outro fator no processo de decisão pelo aborto. Nesta pesquisa, a análise deste dado foi prejudicada pois a profissão ou ocupação das pacientes não foram especificadas em 69,9\% dos prontuários. Quando ocorreu o registro, a ocupação que mais teve realce foi a de trabalho doméstico não remunerado ("do lar"), com 15\% dos casos. Essa ocupação também se destacou em estudos como os de Fonseca et al. (1996) e Schor (1990). 
Tabela 2. Registro do número de abortos em prontuários de mulheres atendidas por abortamento em um Hospital Universitário de Londrina - Paraná, de 2001 a 2005.

\begin{tabular}{lcc}
\hline N. abortos & Freqüência & Porcentagem \\
\hline 1 & 309 & $75,7 \%$ \\
2 & 73 & $17,9 \%$ \\
3 & 15 & $3,7 \%$ \\
4 & 6 & $1,5 \%$ \\
5 & 5 & $1,2 \%$ \\
& & \\
\hline Total & 408 & $100,0 \%$ \\
\hline
\end{tabular}

Conforme mostrado na Tabela 2, em 24,3 $\%$ dos prontuários observaram-se dois ou mais abortamentos. Estudos como os de Schor (1990), Fonseca et al. (1996; 1998) e Murta et al. (2001) mostram a ocorrência significativa de um ou mais abortamentos pela mesma mulher. Os abortamentos de repetição podem estar associados com a falta de conhecimento da mulher quanto ao retorno de sua fertilidade dentro de 15 dias após o abortamento, e essa informação se faz fundamental no pós-abortamento, bem como as orientações contraceptivas. Analisando a ocorrência de apenas um abortamento em $75 \%$ das mulheres, pode-se refletir sobre a possibilidade das mesmas terem "aprendido" a usar o Misoprostol no primeiro abortamento e, uma vez conhecidos os efeitos causados pela medicação, as mesmas se sentem mais seguras para superá-los, sem haver necessidade da busca pelo serviço de saúde.

Tabela 3. Registro relativo à contracepção pós-aborto em prontuário de mulheres atendidas por abortamento em um Hospital Universitário de Londrina - Paraná, de 2001 a 2005.

\begin{tabular}{lcc}
\hline Registro relativo a contracepção pós-aborto & Freqüência & Porcentagem \\
\hline Não há registro & 311 & $76,2 \%$ \\
Prescrição de método & 89 & $21,8 \%$ \\
Encaminhamento a outro serviço & 4 & $1,0 \%$ \\
Orientação a continuar método anterior & 3 & $0,7 \%$ \\
Outro & 1 & $0,3 \%$ \\
& & \\
\hline Total & 408 & $100,0 \%$ \\
\hline
\end{tabular}


Para se evitar a reincidência de abortamentos, é fundamental a orientação contraceptiva por qualquer membro da equipe de saúde e, pela importância desta ação, que fosse registrada no prontuário da paciente. A Tabela 3 mostra que grande parcela $(76,2 \%)$ dos prontuários analisados não apresentou qualquer registro relativo à orientação de contracepção às pacientes no pós-abortamento. É possível que a orientação contraceptiva tenha sido realizada, porém, não registrada.

O Ministério da Saúde recomenda que todas as pacientes no pós-abortamento devem ser orientadas acerca dos métodos contraceptivos para prevenir a gravidez, já que ela estará fértil antes de sua próxima menstruação, podendo engravidar 15 dias após o abortamento (BRASIL, 2003; BRASIL, 2005). Nesta pesquisa, porém, em apenas $21,8 \%$ dos prontuários houve algum tipo de registro que evidenciasse a intenção de prescrever métodos contraceptivos. Em 0,7\% dos prontuários, a orientação foi de continuar utilizando o método anterior. Neste caso, é necessário atentar para a necessidade de ensinar a essa paciente a maneira correta de se utilizar o método, pois, se ela engravidou, provavelmente não o estava utilizando da maneira adequada e as chances de uma futura gravidez se tornam muito grandes. Em 1,0\% dos prontuários havia o registro de encaminhamento para serviços em que a mulher poderia obter o DIU ou a laqueadura. Porém, considerando o tempo de espera na fila do SUS para a realização desses procedimentos, seria necessária a prescrição de outro método contraceptivo que atendesse as necessidades da mulher até que o DIU fosse implantado ou a laqueadura realizada, pois durante esse tempo de espera, a mulher poderia engravidar.

Todas as instruções em relação às orientações anticoncepcionais no pós-abortamento estão registradas em uma Norma Técnica do Ministério da Saúde (BRASIL, 2005). Cabe aos profissionais da saúde buscar o conhecimento sobre as atuais políticas de saúde e as implementarem no dia-dia.
A contra referência para os serviços de atenção básica é fundamental ao acompanhamento das mulheres que abortam. Esses serviços, por meio das Equipes de Saúde da Família, podem reforçar as orientações fornecidas no pós-abortamento, sanar possíveis dúvidas, fornecer os métodos, incentivar a continuidade do uso do método contraceptivo, a fim de prevenir gestações indesejadas e abortamentos (BILLINGS; VERNON, 2007).

Também seria necessária a maior valorização dos registros em prontuários, com a utilização de vocabulário científico, precisão e exatidão, pois eles servem como um elo de comunicação entre a equipe de saúde e auxiliam na avaliação correta dos pacientes, dos cuidados a eles prestados e na realização de pesquisas para melhoria da qualidade da assistência.

\section{Considerações Finais}

Evidencia-se que a orientação contraceptiva no pós-abortamento não tem sido realizada pela equipe de saúde, e é necessária e urgente a capacitação destes profissionais para a qualificação da assistência prestada. Também é fundamental que haja, principalmente nos cursos de Medicina e Enfermagem, a inserção de formação técnica para um atendimento de qualidade no pós-abortamento, que privilegie a orientação contraceptiva como parte fundamental e indispensável deste processo.

É imprescindível que seja implementado o encaminhamento das pacientes atendidas por abortamento para as Unidades Básicas de Saúde a fim de garantir acompanhamento e assistência a essas mulheres de maneira efetiva.

A melhora dos registros hospitalares, com informações corretas, precisas e legíveis é urgente, para que haja conduta adequada, boa comunicação entre as equipes e subsídios para novas pesquisas. 


\section{Referências}

ARILHA, M..; BARBOSA, R. M. Cytotec in Brazil: at least it doesn't kill. Reproductive health matters, London, v. 1, n. 2, p. 41-52, Nov. 1993.

BILlingS, D. L.; VERNON, R., (Ed.). Avances en la atención posaborto en América Latina y el Caribe: investigando, aplicando y expandiendo. México: Population Council, 2007.

BRASIL. Ministério da Saúde. Secretaria de Atenção à Saúde. Departamento de Ações Programáticas Estratégicas. Área Técnica de Saúde da Mulher. Atenção humanizada ao abortamento: norma técnica. Brasília: Ministério da Saúde, 2005.

BRASIL. Ministério da Saúde. Secretaria de Atenção à Saúde. Departamento de Ações Programáticas Estratégicas. Manual dos comitês de mortalidade materna. 3. ed. Brasília: Ministério da Saúde, 2007.

BRASIL. Ministério da Saúde. Secretaria de Políticos de Saúde. Área Técnica de Saúde da Mulher. Parto, abortamento e puerpério: assistência humanizada à mulher. Brasília: Ministério da Saúde, 2001.

COHEN, S. A. Abortion and women of color: the bigger picture. Guttmacher Policy Review, Washington, v. 11, n. 3, p. 2-12, 2008.

COHEN, S. A. Repeat abortion, repeat unintended pregnancy, repeated and misguided government policies. Guttmacher Policy Review, Washington, v. 10, n. 2, p. 8-12, 2007.

CONSELHO REGIONAL DE MEDICINA DO DISTRITO FEDERAL. Prontuário médico do paciente: guia para uso prático. Brasília: Conselho Regional de Medicina, 2006.

CONSÓRCIO DE ATENÇÃO PÓS-ABORTO - APA. Elementos essenciais da atenção pós-aborto: um modelo ampliado e atualizado. APA em Ação, n. 2, Supl. A, set. 2002.

COSTA, R. G. et al. A decisão de abortar: processo e sentimentos envolvidos. Cadernos de Saúde Pública, Rio de Janeiro, v. 11, n. 1, p. 97-105, jan./mar. 1995.

DRAGANOV, P. B.; REICHERT, M. C. F. Avaliação do padrão dos registros de enfermagem em um hospital privado na cidade de São Paulo. Revista de Enfermagem UFPE On Line, Recife, v. 1, n. 1, p. 36-45, 2007.

ESPEJO, X. et al. Adequação do conhecimento sobre métodos anticoncepcionais entre mulheres de Campinas, São Paulo. Revista de Saúde Pública, São Paulo, v. 37, n. 5, p. 583-590, 2003.

FONSECA, W. et al . Características sócio-demográficas, reprodutivas e médicas de mulheres admitidas por aborto em hospital da Região Sul do Brasil. Cadernos de Saúde Pública, Rio de Janeiro, v. 14, n. 2, p. 279-286, abr./jun. 1998.

FONSECA, W. et al. Determinantes do aborto provocado entre mulheres admitidas em hospitais em localidade da região Nordeste do Brasil. Revista de Saúde Pública, São Paulo, v. 30, n. 1, fev. 1996.

GUTTMACHER INSTITUTE. Facts on induced abortion worldwide. Nova York: AGI, 2007.

HADDAD, M. C. L. Qualidade da assistência de enfermagem: o processo de avaliação em hospital universitário público. 2004. 201 f. Tese (Doutorado em Enfermagem Fundamental) - Escola de Enfermagem de Ribeirão Preto, Universidade de São Paulo, Ribeirão Preto, 2004.

HARDY, E. et al. Características atuais associadas à história de aborto provocado. Revista de Saúde Pública, São Paulo, v. 28, n. 1, 1994.

HARVEY, R. A.; CHAMPE, P. C.; MYCEK, J. M. Autacóides e antagonistas. In: HARVEY, R. A. Farmacologia ilustrada. 2. ed. Porto Alegre: Artmed, 1998. p. 419-428.

HENSHAW, S. K.; SING, S.; HAAS, T. La Incidencia del abortamento inducido a nível mundial. Nova York: AGI, 1999.

HOFFMAN, K.; CENTENO, M. A. Um continente entortado (América Latina). Tempo Social, São Paulo, v. 18, n. 2, 2006.

LABBADIA, L. L.; ADAMI, N. P. Avaliação das anotações de enfermagem em prontuários de um hospital universitário. Acta Paulista de Enfermagem, São Paulo, v. 17, n. 1, p. 55-62, 2004.

LANGER, A. El embarazo no deseado: impacto sobre la salud y la sociedad en América Latina y el Caribe. Revista Panamericana de Salud Publica, Washington, v. 11, n. 3, 2002.

LIMA, B. G. C. Mortalidade por causas relacionadas ao aborto no Brasil: declínio e desigualdades espaciais. Revista Panamericana de Salud Publica, Washington, v. 7, n. 3, 2000.

LOUREIRO, D. C.; VIEIRA, E. M.Aborto: conhecimento e opinião de médicos dos serviços de emergência de Ribeirão Preto, São Paulo, Brasil, sobre aspectos éticos e legais. Cadernos de Saúde Pública, Rio de Janeiro, v. 20, n. 3, p. 679-688, maio/jun. 2004.

MARTINS, I. R. et al. Aborto induzido em mulheres de baixa renda: dimensão de um problema. Cadernos de Saúde Pública, Rio de Janeiro, v. 7, n. 2, p. 251-266, abr./ 
jun. 1991.

MOTTA, I. S. $A$ relação interpessoal entre profissionais de saúde e a mulher em abortamento incompleto: o olhar da mulher. Revista Brasileira de Saúde Materno Infantil, Recife, v. 5, n. 2, abr./jun. 2005.

MURTA, E. F. C. et al. $A$ bortamento séptico: identificação de fatores de risco para complicações. Revista Brasileira de Ginecologia e Obstetrícia, Rio de Janeiro, v. 23, n. 3, p. 153-157, abr. 2001.

OLIVEIRA, P. S. et al. Comunicação terapêutica em enfermagem revelada nos depoimentos de pacientes internados em centro de terapia intensiva. Revista Eletrônica de Enfermagem, Goiânia, v. 7, n. 1, p. 54-63, 2005.

ORGANIZACIÓN MUNDIAL DE LA SALUD. Aborto sin riesgos: guia tecnica y de politicas para sistemas de salud. Ginebra: OMS, 2003.

OSIS, M. J. D. et al. Conseqüências do uso de métodos anticoncepcionais na vida das mulheres: o caso da laqueadura tubária. Cadernos de Saúde Pública, Rio de Janeiro, v. 15, n. 3, p. 521-532, jul./set. 1999.

OSIS, M. J. D. et al. Dificuldades para obter informações da população de mulheres sobre aborto ilegal. Revista de Saúde Pública, São Paulo, v. 30, n. 5, p. 444-451, out. 1996.

PÁDUA, A. R. Análise das anotações de enfermagem: uma contribuição à sistematização da assistência de enfermagem. In: SIMPÓSIO BRASILEIRO DE COMUNICAÇÃO EM ENFERMAGEM, 8., 2002, São Paulo. Proceedings... Escola de Enfermagem de Riberão Preto - USP.

PARPINELLI, M. A. et al. Subnotificação da mortalidade materna em Campinas: 1992 a 1994. Revista Brasileira de Ginecologia e Obstetrícia, Rio de Janeiro, v. 22, n. 1, p. 27-32, jan./fev. 2000,

PERES, S. O.; HEILBORN, M. L. Cogitação e prática do aborto entre jovens em contexto de interdição legal: o avesso da gravidez na adolescência. Cadernos de Saúde Pública, Rio de Janeiro, v. 22, n. 7, p.1411-20, jul. 2006.

SALTER, C. et al. $O$ tratamento das complicações pósaborto: uma intervenção para salvar a vida da mulher. Population Reports, Maryland, v. 25, n. 1, p. 1-8, 1997.

SCHOR, N. Investigação sobre ocorrência de aborto em pacientes de hospital de centro urbano do estado de São Paulo, Brasil. Revista de Saúde Pública, São Paulo, v. 24, n. 2, p. 144-151, 1990.

SEDGH, G. et al. Women with an unmet need for contraception in developing countries and their reasons for not using a method. New York: Guttmacher Institute,
2007.

SORRENTINO, S. R.; LEBRAO, M. L. Os abortos no atendimento hospitalar do Estado de São Paulo, 1995. Revista Brasileira de Epidemiologia, São Paulo, v. 1, n. 3,p. 256-267, dez. 1998.

TAVARES, L. S.; LEITE, I. C.; TELlES, F. S. P. Necessidade insatisfeita por métodos anticoncepcionais no Brasil. Revista Brasileira de Epidemiologia, São Paulo, v. 10, n. 2, p. 139-48, jun. 2007.

WEHBE, G.; GALVÃO, C. M. O enfermeiro de unidade de emergência de hospital privado: algumas considerações. Revista Latino-Americana de Enfermagem, Ribeirão Preto, v. 9, n. 2, p. 86-90, mar. 2001.

WORLD HEALTH ORGANIZATION. Unsafe abortion: global and regional estimates of the incidence of unsafe abortion and associated mortality in 2000. 4. ed. Geneva: WHO, 2004.

Recebido em: 30 de janeiro de 2009 Aceito em: 20 de dezembro de 2010 